with persistence of radiological abnormalities in the long-term. The present study shows in a small number of macrocephalic infants followed to young adulthood, an initial orbito-frontal collection of extradural fluid and enlarged subarachnoid space will regress and normalize, in the absence of a co-existing developmental disorder or hydrocephalus. Idiopathic macrocephaly may be considered a normal variant, radiologically, but neuropsychologically, individual variations can be expected, with impairments in visuo-motor skills and attention. Early investigation of cognitive function, and monitoring of behavioral and academic performance may be indicated in infants diagnosed with idiopathic macrocephaly.

\title{
EARLY DETECTION OF CORPUS CALLOSUM GROWTH IMPAIRMENT IN PREMATURE INFANTS
}

The effect of preterm birth on the serial growth of the corpus callosum and cerebellar vermis and the earliest time of detected impairment were studied by cranial ultrasonography in 61 very low birth-weight (VLBW) infants ( $<33$ weeks gestation; $<1500$ gms) admitted to a single regional level III NICU from 1998 to 2000 at Christchurch Hospital, New Zealand. Sonograms were performed twice, $>7$ days apart, in the first 2 weeks of life, at 6 weeks, and at term equivalent. The length of the corpus callosum and vermis was measured on midline sagittal images, and growth rates calculated in $\mathrm{mm}$ per day. The relation between corpus callosum growth rates and neurodevelopmental outcome at 2 years of age was examined. The corpus callosum elongated at a normal rate of $0.21 \mathrm{~mm} /$ day from birth to 2 weeks but slowed to $0.11 \mathrm{~mm} /$ day in weeks $2-6$. A reduction in growth rate of the corpus callosum was detected by 6 weeks in $96 \%$ of infants of 23-33 weeks' gestation, and it persisted to term equivalent in the majority. Some improvement in growth rate of the corpus callosum occurred in $15 \%$ of infants after 6 weeks, but only in VLBW infants born after 28 weeks gestation. Retardation in growth of the vermis was correlated with corpus callosum growth impairment. Motor delay and cerebral palsy at 2 years of age were associated with under-development of the corpus callosum between 2 and 6 weeks after birth. (Anderson NG, Laurent I, Woodward LJ, Inder TE. Detection of impaired growth of the corpus callosum in premature infants. Pediatrics September 2006;118:951-960). (Respond: Dr Nigel G Anderson, Department of Radiology, Christchurch Hospital, Riccarton Avenue, Christchurch 8001, New Zealand).

COMMENT. Corpus callosum white matter injury occurs at the time of birth in almost all infants born prematurely at 23-33 weeks' gestation. The impairment in growth of the corpus callosum and also the vermis of the cerebellum is detectable by bedside ultrasound by 6 weeks of age or earlier, if the damage occurs prenatally. Neuroprotective strategies such as mild hypothermia are best implemented in the NICU as soon after birth as possible. VLBW infants with reduced growth of the corpus callosum detected at 2 to 6 weeks after birth are at increased risk of psychomotor developmental delay and cerebral palsy.

Allometric scaling of brain growth of preterm infants on serial MRIs was used to demonstrate a deficit in cortical surface-area expansion relative to brain volume with increasing prematurity (Kapellou $\mathrm{O}$ et al, reviewed by Allin M. Lancet Neurology Oct $2006 ; 5: 812-813$ ). Slower rates of cortical surface-area growth correlated with developmental delay at 2 years of age, especially in boys. An abnormal subcortical white-matter 
connectivity is proposed, rather than loss of neurons, to explain the neurological and cognitive deficits in preterm babies.

\section{CSF OBSTRUCTION AND MALABSORPTION IN CONGENITAL HYDROCEPHALUS}

The relative contribution of CSF malabsorption and obstruction in three different etiological groups of neonatal high-pressure hydrocephalus (HC) was assessed in a study at University of Bonn, Germany, and University of Groningen, The Netherlands. CSF biomarkers, transforming growth factor beta-1 (TGF B-1), and aminoterminal propeptide of type 1 collagen (PC1NP), indicative of growth factor- and fibrosis-related CSF malabsorption, were assessed and compared in neonates with post-hemorrhagic $\mathrm{HC}(\mathrm{n}=6)$, non-hemorrhagic triventricular HC $(n=4)$ and spina bifida (SB) HC $(n=12)$. CSF interleukin6 (IL-6) cytokines, indicative of inflammation, were low and did not differ between groups. TGF B-1 concentrations were significantly higher in post-hemorrhagic $\mathrm{HC}$ cases (median $355 \mathrm{pg} / \mathrm{ml}$ ) than in SB HC (median 103) and non-hemorrhagic HC (median 120); $\mathrm{p}=0.01$ and 0.03 , respectively. Median CSF PC1NP concentrations were significantly lower in SB HC $(180 \mathrm{ng} / \mathrm{ml})$ than in post-hemorrhagic $\mathrm{HC}(1,060 \mathrm{ng} / \mathrm{ml}) ; \mathrm{p}=0.002$. Neonatal posthemorrhagic $\mathrm{HC}$ is associated with high concentrations of CSF malabsorption-related biomarkers whereas SB and non-hemorrhagic HC have lower concentrations, indicating that CSF obstruction contributes more to the development of these cases than malabsorption. (Heep A, Bartmann P, Stoffel-Wagner B et al. Cerebrospinal fluid obstruction and malabsorption in human neonatal hydrocephaly. Childs Nerv Syst October 2006;22:1249-1255). (Respond: Dr Axel Heep, Department of Neonatology, University of Bonn, Adenauerallee 119, 53113 Bonn, Germany).

COMMENT. High TGF B-1 and PC1NP CSF concentrations in neonatal posthemorrhagic $\mathrm{HC}$ are indicative of a fibrosis-related malabsorption as the cause of the $\mathrm{HC}$, contrasting with relatively low levels of malabsorption biomarkers in SB and nonhemorrhagic triventricular HC. CSF obstruction, rather than malabsorption, plays a major role in the pathogenesis of high-pressure SB and non-hemorrhagic $\mathrm{HC}$.

\section{BRAIN TUMORS}

\section{BONE MINERAL DENSITY REDUCTION FOLLOWING IRRADIATION OF BRAIN TUMORS}

Total body bone mineral density (TBBMD) was measured by X-ray absorptiometry in 46 brain tumor patients aged from 3.8 to 28.7 years (mean $14.9 \mathrm{y}$ ) at a mean of $6.4 \mathrm{y}$ (range 1.4-14.8 y) after end of treatment for brain tumor. Tumors were astrocytic, grade 1, in 25 patients. Tumors were resected in 45 and biopsied in 1. Eleven received chemotherapy. Radiotherapy was cranial in 10 and craniospinal in 5. One third of the patients had low TBBMD ( $\mathrm{z}$ scores $<-2.0$ ). Only combined craniospinal irradiation was significantly associated with low z scores, while cranial irradiation showed a borderline statistical association. (Pietila S, Sievanen H, Ala-Houhala M, et al. Bone mineral density is reduced in 